\title{
Artificial intelligence: what should an intensivist have in mind in the beginning of the new era?
}

\author{
Luiz Alberto Cerqueira Batista Filho \\ Intensive Care Physician, Imed Group Brasil. Condomínio Edifício Reynaldo Raucci, Av. Angélica, 2530 - 9 andar - Bela Vista, São \\ Paulo - SP - 01228-200 - Brazil \\ Correspondence: Luiz Alberto Cerqueira Batista Filho, Rua Afonso Braz, 537, apartamento 82B, Vila Nova Conceição - São Paulo - \\ SP - 04511-011 - Brazil - 04511011; E-mail: luizcerqueira80@gmail.com; Phone: +5511943425200
}

\section{Abstract}

A new era is coming for medicine, and for critical care in particular. The intensive care unit is at the edge of being completely changed by artificial intelligence, and many challenges are ahead of the intensive care physician. This article aims to address the benefits and difficulties that big data will bring to clinicians, and to provide an overview on the subject.

Key words: Big Data; Artificial intelligence; ICU; Critical Care; Black box

Citation: Filho LACB. Artificial intelligence: what should an intensivist have in mind in the beginning of the new era. Anaesth. Pain intensive care 2021;25(1):8-12. DOI: 10.35975/apic.v25i1.1428

Received: 10 December 2020, Reviewed: 3 January 2021, Accepted: 8 January 2021

\section{Introduction}

There is no going back. Artificial Intelligence (AI) will revolutionize healthcare in every aspect, within no more than 10 years. Numerous innovations will come through data generated by physiological monitoring of patients, which is the hallmark of the Intensive Care Unit (ICU). The use of Electronic Health Records (EHR) is of great help in gathering qualitative and quantitative information of patients, making critical care an excellent opportunity for flourishing of automated algorithms. Despite being one of the youngest specialties, Intensive Care Medicine has always been at the forefront, contributing to the development of organizational protocols that changed the care of patients forever. Now, it is time for big data.

\section{Big data and machine learning: An overview}

Since ancient times, physicians have been writing down their experiences on treating patients and alleviating suffering. Countless books and universities were created in an effort to keep, disseminate and structure this knowledge. In the last decades, technology started to play a key role connecting the art of medicine and science, ${ }^{1}$ especially with the adoption of mobile devices. In our days, it is possible to record information about almost every aspect of human life, and healthcare is no exception to this. Continuous physiological monitoring has created high-volume critical care databases, offering substratum for machine learning (ML). Some countries have been developing their own databases in the recent years, ${ }^{2,3,4,5}$ and chances are others will follow in the same direction very soon. The first resource of the kind was the Multiparameter Intelligent Monitoring in Intensive Care (MIMIC), ${ }^{6}$ maintained by the Massachusetts Institute of Technology Laboratory for Computational Physiology. The project is already in its fourth iteration (MIMIC-IV), ${ }^{7}$ containing data from more than 53000 admissions to the Beth Israel Deaconess Medical Center as of $2019 .{ }^{8}$ Another example is the Phillips eICU. ${ }^{9}$ These huge and very complex datasets are colloquially known as big data. 
The mythical figure ${ }^{10}$ of the computer with human-like capabilities, such as communication skills, flexibility, empathy and responsibility, is not a reality yet. ${ }^{11}$ Due to its limitations, it is not expected that AI will replace physicians in the ICU anytime soon. Nonetheless, machines can identify hidden patterns from highvolume datasets through automated algorithms, generating new knowledge, that will build upon human expertise.

ML can be supervised, unsupervised or mixed. ${ }^{12}$ During supervised learning, the machine knows if the patients have the studied disease or not and it looks for patterns within the database to determine which variables will predict the outcome. With unsupervised learning, the outcome is not given to the program. As the final answer is not given, the program looks for patterns within the data, in order to identify different groups. With semi-supervised or mixed ML, the machine will have access to labeled and unlabeled data, and will attempt to categorize patients, and to prognosticate.

Currently, ML is intrinsically related to the use of various statistical techniques, in order to make predictions and decisions based on patterns that will be appreciated from big data analysis. ${ }^{13}$ Some of these advanced statistical techniques are data mining, 14 gradient boosting, ${ }^{15}$ linear regression, ${ }^{16}$ canonical regression ${ }^{17}$ and Bayesian analysis, ${ }^{18}$ amongst others. The objective is applying the revealed frameworks to new data. Predictive models generated by statistical analysis can forecast many outcomes in medicine, allowing clinicians to develop a better-informed decision-making process.

\section{Improving critical care: from protocols to advanced algorithms}

In 1974, William C. Shoemaker wrote an editorial on Protocol Medicine, ${ }^{18}$ elucidating that "routine or patient protocols are useful means to standardize care, to facilitate completeness of services, and to evaluate both patient's progress and the therapeutic efficacy of the program. It is also an educational tool". Meade and Ely emphasized in 2002 that "the use of protocols has yielded some of the most important improvements in patient mortality and morbidity. ${ }^{20}$ Now, in the third decade of the new millennium, advanced algorithms will be responsible for boosting intensive care, and medicine in a broader sense. Machine learning is already being used, amongst other features, to prognosticate patient outcomes, ${ }^{21}$ to predict prolonged ventilation and tracheostomy, ${ }^{21}$ acute kidney injury, ${ }^{23}$ hypotension in perioperative patients, ${ }^{24}$ pressure injury, ${ }^{25}$ volume responsiveness ${ }^{26}$ and the need of critical care in prehospital emergency medical services. ${ }^{27}$ Depth of sedation is being accessed by collecting data from continuous electroencephalography. ${ }^{28}$ Pain prediction has been evaluated from brain imaging, using functional magnetic resonance analysis of human volunteers exposed to painful and non-painful thermal stimuli. ${ }^{29}$ The multivariate machine-learning models built from brain imaging and autonomic activity can predict clinical pain more accurately than traditional analysis of individual brain regions associated with nociception. In a few years, it might be possible to predict perioperative pain before patients are admitted to the ICU.

In the near future, physicians will regularly make clinical decisions strengthened by machines. ${ }^{30}$ Choosing the most suitable algorithm for each situation will be a challenge, keeping in mind that data issues will occur. ${ }^{31} \mathrm{We}$ will need distinct and validated algorithms for different populations, since data will vary from one to another. Therefore, quality and quantity of data is vital. The necessity for new datasets will come to light, making individualization of algorithms essential. Cellular and molecular biology will be shifted into clinical practice by adding "omic" data to the equation. ${ }^{32}$ Genomics, epigenomics, transcriptomics, metabolomics and proteonomics will probably play a significant role in sepsis, by subtyping the disease and taking individualization to a whole new level. ${ }^{33}$ Aiming for a patient-tailored treatment will be the goal. The path towards a precision medicine is already being traced.

Unprecedented occasion for expanding databases at developing countries are at the doorstep, since these will be the last to enter the new era. Endless opportunities for research are at hand, as medicine is about to be rediscovered.

\section{The hospital without walls}

In 2002, Ken Hillman anticipated a bigger role for the critical care physician outside the ICU. In the paper 
"Critical Care without Walls" 34 he foresaw the establishment of the fast response teams, offering rapid recognition and treatment to the critically ill patients at any ward of the hospital. Today, we are heading into an era of expanding the very limits of the hospitals. Machine learning is turning wearable intelligent monitoring devices into powerful tools that will create an explosion of clinical data, inside and outside the healthcare institutions. We already know that critical illness is preceded by undetected changes in vital signs over a period of hours. ${ }^{35}$ Hospitals and clinics will have departments dedicated to data evaluation from patients in their homes, using algorithms to predict the necessity of admission. Data oriented mobile apps will give support to patients, becoming virtual health assistants, and reducing length of stay in institutions, since much of the problems will be remotely solved. These predictive models will provide a more efficient use of resources, as well as facilitate prioritization and personalization of services. ${ }^{36}$ Palliative care and postICU patients will have the chance to spend more time at their homes with their families. Data analysis will predict the chance of readmission and clinical complications.

Beyond any doubt, privacy preservation will become a major concern for healthcare institutions, since personal medical data will be continually shared. Encryption $^{37}$ and de-identification ${ }^{38}$ of data will be necessary, in order to protect privacy at minimal cost to innovation. Although confidentiality is essential in the context of medical records, it is important to keep in mind that fragmented and de-identified data imposes significant technological and economic hurdles to the development of AI. ${ }^{39}$ To determine the appropriate balance on this matter will be a key issue in the future.

\section{The black box problem: a troubled frontier between the human mind and artificial intelligence}

Sooner than we think, physicians will have the responsibility of choosing algorithms during their clinical practice, aiming to offer the best solutions to patients' problems. Considering that AI will become integrated into the physician`s workflow, an important question arises: will the majority of doctors be qualified enough to make such decisions?
The "Black Box" problem refers to the difficulty that the human brain contends with understanding how AI agents make clinical decisions. ${ }^{40}$ Sometimes, it will be difficult to follow the intricated ways that lead the program to a clinical decision. Some doctors will be resistant to the use of $\mathrm{AI}$, and many will have a hard time developing a taste for technological affairs. However, disregard of the fundamental concepts of ML might lead to hesitation and mistakes, the consequences of which can be fatal on a critical care environment. Although the idea of bearing an intelligent machine at the hospital is exactly not having to be an expert on computational science, assimilating primary notions will be very important.

Extreme caution is advisable on the implementation of AI, as the clinical staff and the multidisciplinary team must have technological proficiency. Clinicians will have to be confident of these new resources, so they can build trust in patients and their families. Surveys made with the public have shown that most people believe that AI will have a positive impact in healthcare. ${ }^{41}$ Yet, people are also less likely to use medical services that are known to use AI. ${ }^{42}$

New disciplines will emerge at medical school, in order to provide physicians with the capacity of dealing with the new reality. Medical residency is about to be redesigned as well.

\section{Conclusion}

In the next ten years, AI will be a groundbreaking innovation, empowering the intensive care physician. A lack of time is a common problem that these professionals face, especially in developing countries, where resources are scarce. Algorithms will allow doctors to spend more time at bedside and with the patients' families, exerting their indispensable human skills. Nonetheless, every benefit comes with a duty. Keeping an updated knowledge on the technological transformations will be a heavy task. On the other hand, a new world of possibilities is closing-in, and the limits are yet to be discovered.

\section{Conflict of interest}

None declared by the author.

\section{Author's contribution}

LACBF is the sole author of the manuscript 


\section{References}

1. Meskó B. The Real Era of the Art of Medicine Begins with Artificial Intelligence. J Med Internet Res. 2019 Nov 18;21(11):e16295. [PubMed] DOI: $10.2196 / 16295$

2. Qi S, Mao Z, Hu X, Liu C, Kang H, Zhou F. [Introduction of critical care database based on specialized information systems: a model of critical care medicine database in large Level III Grade A hospital]. Zhonghua Wei Zhong Bing Ji Jiu Yi Xue. 2020 Jun;32(6):743-749. Chinese. [PubMed] DOI: 10.3760/cma.j.cn12143020200520-00393

3. Damian MS, Ben-Shlomo Y, Howard R, Harrison DA Admission patterns and survival from status epilepticus in critical care in the UK: an analysis of the Intensive Care National Audit and Research Centre Case Mix Programme database. Eur J Neurol. 2020 Mar;27(3):557-564. [PubMed] DOI: 10.1111/ene.14106.

4. Irie $\mathrm{H}$, Okamoto $\mathrm{H}$, Uchino $\mathrm{S}$, Endo $\mathrm{H}$, Uchida $\mathrm{M}$, Kawasaki $T$, et al.; JIPAD Working Group in the Japanese Society of Intensive Care Medicine. The Japanese Intensive care PAtient Database (JIPAD): A national intensive care unit registry in Japan. J Crit Care. 2020 Feb;55:86-94. [PubMed] DOI: 10.1016/j.jcrc.2019.09.004.

5. Jackson Chornenki N, Liaw P, Bagshaw S, Burns K, Dodek $\mathrm{P}$, English $\mathrm{S}$, et al.; Canadian Critical Care Trials Group (CCCTG) and Canadian Critical Care Translational Biology Group (CCCTBG). Data initiatives supporting critical care research and quality improvement in Canada: an environmental scan and narrative review. Can J Anaesth. 2020 Apr;67(4):475484. English. [PubMed] DOI: 10.1007/s12630-02001571-1

6. Johnson AE, Pollard TJ, Shen L, Lehman LW, Feng M, Ghassemi M, et al. MIMIC-III, a freely accessible critical care database. Sci Data. 2016 May 24;3:160035. [PubMed] DOI: $10.1038 /$ sdata.2016.35

PMCID: PMC4878278.

7. MIMIC-IV. 2020. MIMIC-IV Documentation. [online] Available at: <https://mimic-iv. mit.edu/docs/> [Accessed 5 December 2020].

8. Pollard TJ, Johnson AEW, Raffa JD, Celi LA, Mark RG, Badawi O. The elCU Collaborative Research Database, a freely available multi-center database for critical care research. Sci Data. 2018 Sep 11;5:180178. [PubMed] PMCID: PMC6132188. DOI: 10.1038/sdata.2018.178

9. Cosgriff CV, Celi LA, Stone DJ. Critical Care, Critical Data. Biomed Eng Comput Biol. 2019 Jun 12;10:1179597219856564

PMCID: PMC6563388.

DOI: $10.1177 / 1179597219856564$

10. Natale S, Ballatore A. Imagining the thinking machine: Technological myths and the rise of artificial intelligence. Convergence. 2020;26(1):3-18. DOI: 10.1177/1354856517715164

11. Komorowski M. Artificial intelligence in intensive care: are we there yet? Intensive Care Med. 2019
Sep;45(9):1298-1300. [PubMed] DOI: 10.1007/s00134019-05662-6

12. Handelman GS, Kok HK, Chandra RV, Razavi AH, Huang S, Brooks M, et al. Peering Into the Black Box of Artificial Intelligence: Evaluation Metrics of Machine Learning Methods. AJR Am J Roentgenol. 2019 Jan;212(1):38-43. [PubMed] DOl: 10.2214/AJR.18.20224

13. Erratum in: AJR Am J Roentgenol. 2019 Feb;212(2):479. PMID: 30332290.

14. Rush B, Celi LA, Stone DJ. Applying machine learning to continuously monitored physiological data. J Clin Monit Comput. 2019 Oct;33(5):887-893. [PubMed] PMCID: PMC6511324. DOI: $10.1007 / s 10877-018-$ 0219-Z

15. Mellor JC, Stone MA, Keane J. Application of Data Mining to "Big Data" Acquired in Audiology: Principles and Potential. Trends Hear. 2018 JanDec;22:2331216518776817. [PubMed] PMCID: PMC6022814. DOI: $10.1177 / 2331216518776817$

16. Zhang Z, Zhao Y, Canes A, Steinberg D, Lyashevska O; written on behalf of AME Big-Data Clinical Trial Collaborative Group. Predictive analytics with gradient boosting in clinical medicine. Ann Transl Med. 2019 Apr;7(7):152. [PubMed] PMCID: PMC6511546. DOI: 10.21037/atm.2019.03.29

17. Marill KA. Advanced statistics: linear regression, part II: multiple linear regression. Acad Emerg Med. 2004 Jan;11(1):94-102. [PubMed] DOI: 10.1197/j.aem.2003.09.006

18. Luo C, Liu J, Dey DK, Chen K. Canonical variate regression. Biostatistics. 2016 Jul;17(3):468-83. [PubMed] PMCID: PMC5006412.

DOI: 10.1093/biostatistics/kxw001

19. Kruschke JK, Liddell TM. Bayesian data analysis for newcomers. Psychon Bull Rev. 2018 Feb;25(1):155177. [PubMed] DOI: 10.3758/s13423-017-1272-1

20. Shoemaker WC. Editorial: Protocol medicine. Crit Care Med. $1974 \quad$ Sep-Oct;2(5):279. [PubMed] DOI: $10.1097 / 00003246-197409000-00010$

21. Meade MO, Ely EW. Protocols to improve the care of critically ill pediatric and adult patients. JAMA. $2002 \mathrm{Nov}$ 27;288(20):2601-3. [PubMed] DOI: 10.1001/jama.288.20.2601

22. Meiring $\mathrm{C}$, Dixit A, Harris S, MacCallum NS, Brealey DA, Watkinson PJ, et al. Optimal intensive care outcome prediction over time using machine learning. PLoS One. 2018 Nov 14;13(11):e0206862. [PubMed] PMCID: PMC6241126. DOI: 10.1371 /journal.pone. 0206862

23. Parreco J, Hidalgo A, Parks JJ, Kozol R, Rattan R. Using artificial intelligence to predict prolonged mechanical ventilation and tracheostomy placement. J Surg Res. 2018 Aug;228:179-187. [PubMed] DOI: 10.1016/j.jss.2018.03.028 
24. Gameiro J, Branco T, Lopes JA. Artificial Intelligence in Acute Kidney Injury Risk Prediction. J Clin Med. 2020 Mar 3;9(3):678. [PubMed] PMCID: PMC7141311. DOI: $10.3390 / \mathrm{jcm} 9030678$

25. Saugel B, Kouz K, Hoppe P, Maheshwari K, Scheeren TWL. Predicting hypotension in perioperative and intensive care medicine. Best Pract Res Clin Anaesthesiol. 2019 Jun;33(2):189-197. [PubMed] DOl: 10.1016/j.bpa.2019.04.001

26. Alderden J, Pepper GA, Wilson A, Whitney JD, Richardson S, Butcher R, et al. Predicting Pressure Injury in Critical Care Patients: A Machine-Learning Model. Am J Crit Care. 2018 Nov;27(6):461-468. [PubMed] DOI: $\underline{10.4037 / a j c c 2018525}$

PMCID: PMC6247790.

27. Zhang Z, Ho KM, Hong Y. Machine learning for the prediction of volume responsiveness in patients with oliguric acute kidney injury in critical care. Crit Care. 2019 Apr 8;23(1):112. [PubMed] PMCID: PMC6454725. DOI: 10.1186/s13054-019-2411-z

28. Kang DY, Cho KJ, Kwon O, Kwon JM, Jeon KH, Park H et al. Artificial intelligence algorithm to predict the need for critical care in prehospital emergency medical services. Scand J Trauma Resusc Emerg Med. 2020 Mar 4;28(1):17. [PubMed] PMCID: PMC7057604. DOI: $10.1186 / \mathrm{s} 13049-020-0713-4$

29. Shalbaf A, Saffar M, Sleigh JW, Shalbaf R. Monitoring the Depth of Anesthesia Using a New Adaptive Neurofuzzy System. IEEE J Biomed Health Inform. 2018 May;22(3):671-677. [PubMed] DOI: 10.1109/JBHI.2017.2709841

30. Lee J, Mawla I, Kim J, Loggia ML, Ortiz A, Jung C, et al Machine learning-based prediction of clinical pain using multimodal neuroimaging and autonomic metrics. Pain. 2019 Mar;160(3):550-560. [PubMed] PMCID: PMC6377310. DOI: 10.1097/i.pain.0000000000001417

31. Magrabi F, Ammenwerth E, McNair JB, De Keizer NF, Hyppönen $\mathrm{H}$, Nykänen $\mathrm{P}$, et al. Artificial Intelligence in Clinical Decision Support: Challenges for Evaluating Al and Practical Implications. Yearb Med Inform. 2019 Aug;28(1):128-134. [PubMed] PMCID: PMC6697499. DOI: $10.1055 / \mathrm{s}-0039-1677903$

32. Carra G, Salluh JIF, da Silva Ramos FJ, Meyfroidt G. Data-driven ICU management: Using Big Data and algorithms to improve outcomes. J Crit Care. 2020 Sep 9;60:300-304. [PubMed] DOl: 10.1016/j.jcrc.2020.09.002

33. Subramanian I, Verma S, Kumar S, Jere A, Anamika K. Multi-omics Data Integration, Interpretation, and Its
Application. Bioinform Biol Insights. 2020 Jan 31;14:1177932219899051

PMCID: PMC7003173.

DOI: $10 . \overline{1177 / 1177932219899051}$

34. Itenov TS, Murray DD, Jensen JUS. Sepsis: Personalized Medicine Utilizing 'Omic' Technologies-A Paradigm Shift? Healthcare (Basel). 2018 Sep 7:6(3):111. [PubMed] PMCID: PMC6163606. DOI: $10.3390 /$ healthcare6030111

35. Hillman K. Critical care without walls. Curr Opin Crit Care. 2002 Dec;8(6):594-9. [PubMed] DOI: 10.1097/00075198-200212000-00019

36. Goldhill DR, White SA, Sumner A. Physiological values and procedures in the $24 \mathrm{~h}$ before ICU admission from the ward. Anaesthesia. 1999 Jun;54(6):529-34. [PubMed] DOI: 10.1046/j.1365-2044.1999.00837.x

37. Reddy S, Fox J, Purohit MP. Artificial intelligenceenabled healthcare delivery. J R Soc Med. 2019 Jan;112(1):22-28. [PubMed] DOI: 10.1177/0141076818815510

38. Vizitiu A, Nita $\mathrm{Cl}$, Puiu A, Suciu C, Itu LM. PrivacyPreserving Artificial Intelligence: Application to Precision Medicine. Annu Int Conf IEEE Eng Med Biol Soc. 2019 Jul;2019:6498-6504. [PubMed] DOI: 10.1109/EMBC.2019.8857960

39. Kayaalp M. Patient Privacy in the Era of Big Data. Balkan Med J. 2018 Jan 20;35(1):8-17. [PubMed] PMCID: PMC5820452

DOI: 10.4274/balkanmedj.2017.0966

40. Price WN 2nd, Cohen IG. Privacy in the age of medical big data. Nat Med. 2019 Jan;25(1):37-43. [PubMed] PMCID: PMC6376961.

DOI: $\underline{10.1038 / \mathrm{s} 41591-018-0272-7}$

41. London AJ. Artificial Intelligence and Black-Box Medical Decisions: Accuracy versus Explainability. Hastings Cent Rep. 2019 Jan:49(1):15-21. [PubMed] DOI: 10.1002/hast.973

42. Canadian Medical Association. 2019. The Future of Connected Health Care: Reporting Canadians' Perspectives on the Health Care System [online]. Available

at: $<$ <ttps://www.cma.ca/sites/default/files/pdf/MediaReleases/The-Future-of-Connected-Healthcaree.pdf>. [Accessed 8 December 2020].

43. Longoni $\mathrm{C}$, Bonezzi $\mathrm{A}$, Morewedge CK. Resistance to medical artificial intelligence. J Consum Res. 2019;46(4):629-650. 